\title{
Diseño de un nuevo capitel para invernaderos multitúnel
}

\section{Desing of a new multispan greenhouse capital}

\author{
J. Vázquez $z^{(*)}$, J. Pérez $z^{(*)}$, A. J. Callejón ${ }^{(*)}, \underline{A}^{\text {A Carreño }}{ }^{(*)}$
}

\section{RESUMEN}

Se presenta un trabajo de investigación que ha tratado de diseñar un nuevo capitel para invernaderos multitúnel, compatible con el procedimiento constructivo de invernadero de cubierta elevable. Se ha empleado el método de los elementos finitos (M.E.F.) para obtener una visión más cercana de las deficiencias estructurales del capitel base, así como para validar las propuestas de mejora establecidas con la definición geométrica del nuevo capitel. Los resultados han puesto de manifiesto que el desarrollo, material y dimensiones del nuevo capitel solventan las carencias estructurales del capitel base, todo ello conforme a lo establecido en la norma de invernaderos EN 13031-1. Se espera que los resultados presentados sean útiles para el desarrollo de futuras investigaciones, así como para la industria de la construcción de invernaderos.

845-15

Palabras clave: Diseño, capitel, invernadero, multitúnel, Método de Elementos Finitos (M.E.F.).

\section{SUMMARY}

The present paper shows a research where a new capital for multispan greenhouses has been designed with the requirement of compatibility with the construction procedure consisting on raising the greenhouses roof. To obtain a better view of the base capital structural deficiencies, and to verify the efficiency of adopted measures with the new capital geometrical resizing, the Finite Element Method (F.E.M.) has been used. The results shows that the new capital geometry, material and dimensions solve structural deficiencies, taken into account the European greenhouses standard EN-13031-1. It is expected that results showed in the present paper will be useful for new researches, and also for greenhouses construction industry.

Key Words: design, capital, greenhouse, multispan, Finite Elements Method (F.E.M.). 
1. Procedimiento constructivo tradicional.

\section{INTRODUCCIÓN}

Los invernaderos se pueden definir como estructuras usadas para el cultivo y/o protección de plantas y cosechas, las cuales optimizan la transmisión de radiación solar bajo condiciones controladas para mejorar el entorno del cultivo y cuyas dimensiones posibilitan el trabajo de las personas en su interior (1).

España es el país más importante en el uso de invernaderos de cubierta plástica de la Unión Europea (2) existiendo, en dicha región, unas 45.000 ha (3), especialmente a lo largo de la costa mediterránea, localizándose la mayor concentración en la provincia de Almería (4) con 25.983 ha de invernaderos (5).

En dicha región, los invernaderos se destinan al cultivo de hortalizas (6), construyéndose con estructuras ligeras y de bajo coste, adaptadas a los requerimientos climáticos (7), siendo el modelo estructural tradicionalmente empleado el denominado "tipo parral", aunque en estas últimas décadas está siendo sustituido por otros más perfeccionados, como los llamados "raspa y amagado" y los invernaderos "industriales", que posibilitan un mejor control climático así como posibilidades de automatización (8).

El invernadero industrial más característico en España es el denominado multitúnel (9), tratándose de una estructura modular de cubierta curva, erigida en su totalidad con perfiles conformados en frío (4).

En Europa existe una normativa que define los principios generales y requisitos de resistencia mecánica y estabilidad, estado de servicio y durabilidad para el proyecto y construcción de invernaderos multitúnel (1). Algunos autores (10), recomiendan su aplicación para el diseño y dimensionado de dichas estructuras. Así, se estudia la problemática de su aplicación en invernaderos multitúnel ubicados en Almería (11) y se plantea una serie de propuestas de mejora estructurales en dichos invernaderos para verificar su cumplimiento (12). La norma de invernaderos también ha sido empleada para estudiar la estabilidad estructural de un invernadero multitúnel con la aplicación de cargas progresivas hasta alcanzar su colapso estructural (13).

Por otra parte, se hace manifiesta la presencia de investigaciones orientadas a conocer la aerodinámica de invernaderos multitúnel (14-17) así como de otras (9) en las que se emplean técnicas de simulación en 3D para la modelización de dichas estructuras con la finalidad de resolver problemas en los procesos de fabricación industrial, mientras que en materia de seguridad las investigaciones son escasas, pese a que se trata de un sector poco tecnificado y en el que no se suelen utilizar técnicas preventivas de seguridad en el trabajo (18) (Figura 1).

Referente a este aspecto, el sector de la construcción de invernaderos en Almería presenta una siniestralidad similar al sector general de la construcción (19), destacando que el 23\% de los siniestros se producen por caídas en altura, asociándoles una mayor severidad del daño (20). Para paliar dicha situación, se plantea un nuevo procedimiento constructivo capaz de controlar, mediante modificaciones en fase de diseño, los riesgos de caída en altura $(20,21)$, cuyo principio concuerda con las investigaciones desarrolladas por diversos autores (22-25).

Este nuevo procedimiento constructivo se fundamenta en el montaje del conjunto de la estructura de cubierta a nivel de suelo y posteriormente su elevación, hasta alcanzar su posición definitiva, empleando los pilares como guías de desplazamiento (Figura 2). Experimentalmente, se ha logrado verificar la idoneidad del sistema, cifrando en un $40 \%$ la reducción de horas de trabajo en altura $y$, consecuentemente, con una disminución de los riesgos derivados en un porcentaje similar.

Una de las piezas clave de este nuevo procedimiento es el capitel. Se trata de un elemento metálico que actúa como nudo articulado enlazando el conjunto de los elementos de cubierta que confluyen en cabeza de pilar (arco, tirante de cercha y canalón), transmitiendo el conjunto de esfuerzos a los pilares. El capitel diseñado para el procedimiento constructivo de invernadero constructivo de invernadero de cubierta elevable se diferencia del resto de capiteles para invernaderos porque se compone de cuatro piezas simétricas o semicapiteles ensamblados entre si una vez alcanzada su posición definitiva en altura $(20,21)$, lo cual permite la elevación independiente de los túneles y su posterior fijación.

A partir de los muestreos visuales realizados, surge la necesidad de rediseñar este elemento ya que se detecta la presencia de fisuras y/o deformaciones en la zona destinada a la recepción del arco de cubierta.

Numerosas investigaciones han empleado técnicas de simulación numérica para estudiar nudos estructurales. Así, se emplea el método de los elementos finitos para estudiar uniones soldadas, identificando la presencia de gradientes de tensiones en las zonas de aporte de soldadura, justificando de esta forma la posterior presencia de fisuras en dichas regiones (26). Por otro lado, también se emplean dichos métodos numéricos para 
estudiar el comportamiento estructural de uniones atornilladas con diferentes perfiles de acero (27), mientras que otros autores tratan de conocer la distribución de tensiones en una unión por ensamble para estructuras de madera, planteando la posible optimización geométrica del ensamble estudiado (28).

También es destacable la presencia de trabajos específicos sobre diseño de uniones para estructuras ligeras, siendo mencionable el diseño de una unión articulada para láminas de acero denominada Rosette (29), o el empleo del análisis de elementos finitos para proponer recomendaciones de diseño sobre acartelamientos en acero conformado en frío (30).

Por otro lado, han sido estudiados los diferentes sistemas de enlace para perfiles conformados en frío, advirtiendo de la necesidad de realizar futuras investigaciones sobre conexiones basadas en técnicas de encaje óptimo (31), siendo éste el objeto de la investigación desarrollada.

\section{OBJETIVOS}

Dada la ausencia de trabajos centrados en el comportamiento de nudos capitel para construcción de invernaderos, se desprende la necesidad de profundizar en su estudio.

Se pretende estudiar por el método de los elementos finitos el comportamiento estructural del capitel diseñado para el procedimiento constructivo de invernadero de cubierta elevable y así, poder justificar las deficiencias estructurales detectadas experimentalmente. Debido a ellas, se plantea el diseño de un nuevo capitel que será validado con un procedimiento de análisis numérico similar al empleado con el capitel base.

\section{MATERIALES Y MÉTODOS}

Para estudiar la estabilidad estructural de los nudos capitel, se requiere el modelado tridimensional de las articulaciones, continuando con la disposición de las fuerzas que actúan sobre ellas.

Para el conocimiento de los esfuerzos que actúan sobre el capitel, es necesario el análisis numérico del comportamiento estructural de un invernadero industrial. De esta forma es posible seleccionar el nudo capitel más limitativo para las diferentes hipótesis combinatorias de cálculo a considerar.

\subsection{Análisis M.E.F. de estructura de invernadero industrial}

Se ha diseñado un invernadero industrial en su variante tipo multitúnel, dado que se trata de la tipología más característica que presentan dichas estructuras en España (9), siendo este mismo criterio el que se ha empleado para definir la geometría del invernadero a estudiar. De esta forma, se ha modelado una estructura constituida por pórticos de $8 \mathrm{~m}$ de

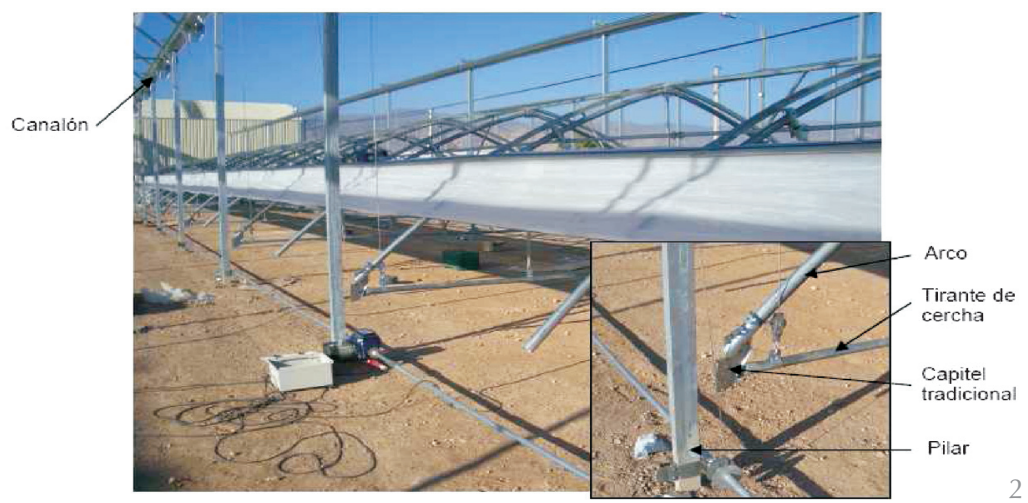

luz dispuestos cada $5 \mathrm{~m}$, con arcos flotantes intercalados cada $5 \mathrm{~m}$, ubicando los pilares perimetrales cada 2,5 m de longitud. La altura de pilares se modela con $4,5 \mathrm{~m}$ y la altura de cumbrera con 6 m (Figura 3).

La modelización se ha realizado teniendo en cuenta las siguientes premisas:

- Todos los nudos de la estructura se consideran articulados, con libertad de giro en el espacio.

- Todos los pilares que componen la estructura se consideran empotrados en su base y articulados en cabeza.

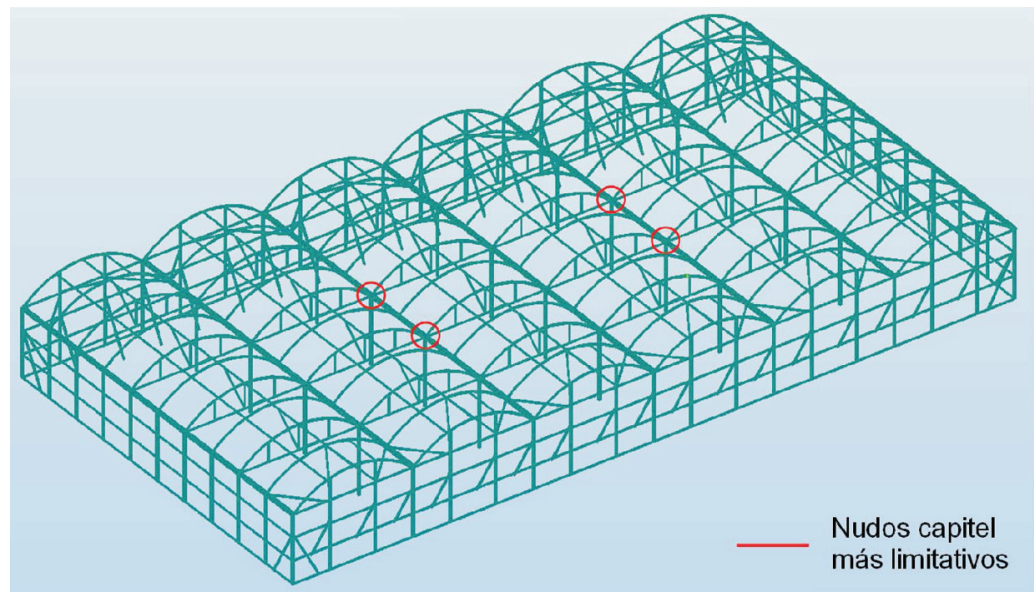

- Los arcos se consideran doblemente articulados en sus apoyos, cabeza de pilares.

2. Constructivo de invernadero de cubierta elevable.

- La sección transversal de los arcos se considera constante, sin variación a lo largo de su desarrollo.

- En el plano perpendicular a los arcos, se ha considerado el arriostramiento que originan elementos horizontales tales 


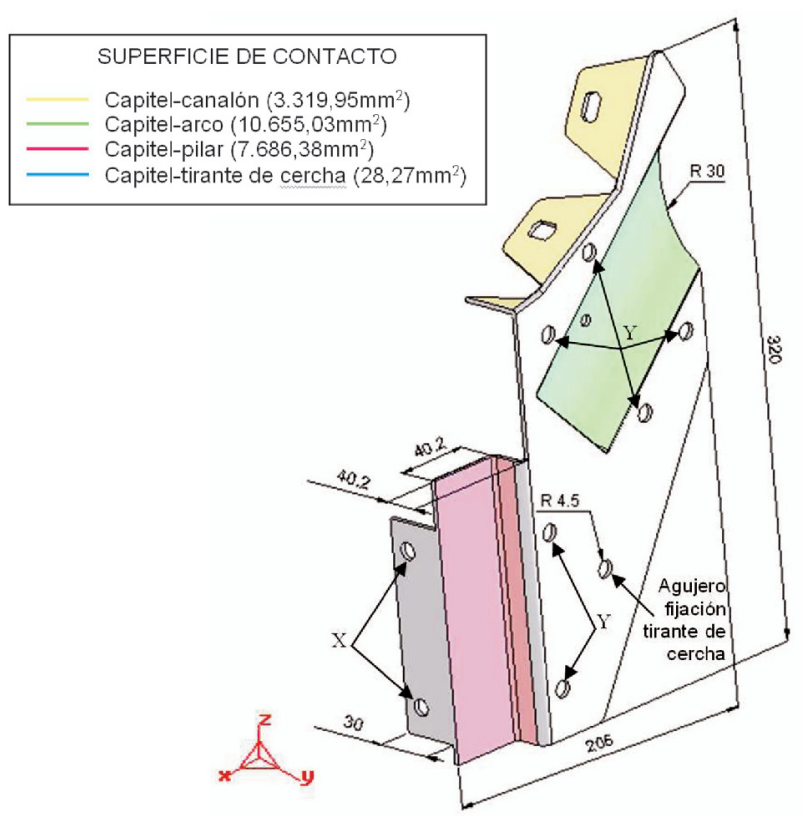

4. Capitel base estudiado. como correas, ya sean cenitales o laterales, y canalón.

- Se han empleado los perfiles de acero habituales en dichos invernaderos en el litoral español ( $\operatorname{arcos} 60 \times 1,5 \mathrm{~mm}$, tirantes de cercha y montantes $32 \times 1,5 \mathrm{~mm}$, pilares $80 \times 80 \times 2 \mathrm{~mm}$, pies de amigo $60 \times 1,5 \mathrm{~mm}$ y canalón: desarrollo $430 \mathrm{~mm}$ y espesor $1,8 \mathrm{~mm}$ ).

Análisis preliminares fueron realizados para decidir la superficie del invernadero a estudiar de forma que contemplase, por un lado, la mayor precisión en los cálculos y, por otra parte, un tiempo de computación razonable.

Así, se logra concretar que una estructura de superficie $1.200 \mathrm{~m}^{2}(48 \mathrm{~m} \times 25 \mathrm{~m})$ resulta ser representativa de otras de mayor extensión, siendo la superficie media que presentan los invernaderos en la provincia de Almería de $7.300 \mathrm{~m}^{2}(32)$.

Una vez definida la geometría del invernadero a estudiar, la siguiente etapa comprende la definición de las cargas que actuaban sobre éste. Dada la necesidad de concretar una zona para la cuantificación de las cargas, se ha supuesto la ubicación de la estructura en la provincia de Almería, puesto que se trata de la región donde se ubica la mayor concentración de invernaderos de cubierta plástica en la Unión Europea (4).

En concreto, se ha supuesto su ubicación en suelos de categoría II, Zona Eólica X y en ausencia de colinas aisladas o escarpaduras, atendiendo a lo establecido en la norma UNE sobre acciones del viento (33).

Los valores característicos de la acciones se han cifrado para una estructura de inverna- dero de cubierta plástica con 10 años de vida útil, de clase B-10, según las normas (1, 33-35). Los coeficientes de presión han sido estudiados para la hipótesis de dirección de viento $0^{\circ}$, viento en el plano del pórtico, dado que resulta la condición más desfavorable (12).

Posteriormente, se ha calculado el invernadero atendiendo a la comprobación de Estados Límites Últimos, considerando para tal fin las combinaciones de acciones, así como los coeficientes parciales y de combinación dictaminados por la norma europea de invernaderos (1).

De esta forma se ha logrado conocer los valores numéricos de las fuerzas y momentos que actúan sobre el conjunto de elementos que constituyen la estructura del invernadero, pudiendo así concretar el nudo más desfavorable para las distintas hipótesis combinatorias anteriormente mencionadas.

\subsection{Análisis M.E.F. del capitel base}

Con el capitel base, el nudo se ejecuta con el ensamblado de cuatro piezas simétricas en cabeza de pilar. De esta forma, cada uno de los dos arcos que son enlazados por la articulación, son fijados por encaje entre dos capiteles, acoplándose el canalón en el recinto definido por los capiteles en su parte superior $y$, el tirante de cercha, en el agujero practicado para tal fin (Figura 4). En todo caso, las uniones se efectúan mediante tornillería.

Dado el carácter simétrico de los capiteles empleados, con dos planos de simetría, el análisis M.E.F. se inicia con la definición geométrica tridimensional de una de las piezas, modelando de esta forma un capitel de dimensiones genéricas $206 \mathrm{~mm}$ de longitud, $320 \mathrm{~mm}$ de altura y 70,2 $\mathrm{mm}$ de anchura, con $2 \mathrm{~mm}$ de espesor (Figura 4).

A continuación, se analizan las superficies de contacto del capitel con respecto al conjunto de los elementos que enlaza (canalón, arco, tirante de cercha y pilar), con el empleo del modelo tridimensional realizado. Para tal fin, se delimitan dichas regiones y cuantifica su superficie (Figura 4). Posteriormente, el conjunto de los esfuerzos, axiales y cortantes, son aplicados superficialmente a las zonas de contacto bajo el supuesto de la existencia de una distribución de carga uniforme.

De todos los agujeros que presenta el capitel, únicamente recibe la aplicación de cargas el encargado de fijar el tirante de cercha. Dado que el tirante de cercha se fija a dos capiteles ensamblados, se ha supuesto una 
distribución equitativa de esfuerzos en ambos capiteles, siendo, por tanto, la carga que percibe uno de ellos la mitad de la total.

Razonamientos semejantes se han realizado para determinar las cargas transmitidas en las superficies de contacto del canalón y el arco. Por otra parte, se aplican condiciones de coacción en los tres grados de libertad en las superficies de contacto del capitel con respecto al pilar y condiciones de coacción en los ejes $\mathrm{X}$ e $\mathrm{Y}$ en los agujeros denotados como $\mathrm{X}$ e $\mathrm{Y}$ respectivamente en la Figura 4, dado que la articulación se constituye por cuatro capiteles simétricos ensamblados mediante tornillería.

Una vez definidas las condiciones de estudio, se procede con la simulación numérica y el análisis de resultados, con el estudio del estado tensional y de los desplazamientos.

\subsection{Análisis M.E.F. del nuevo capitel diseñado}

Siendo conocidas las deficiencias estructurales en materia de tensión y deformación del capitel base, se plantean una serie de propuestas de mejora concretadas con una nueva geometría de capitel (Figura 5). El nuevo capitel presenta unas dimensiones genéricas de 295 $\mathrm{mm}$ de longitud, $274 \mathrm{~mm}$ de altura y $44,5 \mathrm{~mm}$ de anchura, con espesor $2 \mathrm{~mm}$.

Con el empleo del nuevo capitel, la articulación se constituye con dos piezas homólogas ensambladas mediante tornillería, fijando los arcos al capitel mediante las correspondientes abrazaderas de unión (Figura 6).

Con un procedimiento de trabajo análogo al desarrollado en el capitel base, se delimitan y cuantifican sobre el modelo 3D las superficies de contacto del capitel con respecto a los elementos que enlaza (Figura 5), continuando con la aplicación de los correspondientes esfuerzos. De nuevo, los esfuerzos son aplicados superficialmente a las zonas de contacto bajo el supuesto de la existencia de una distribución de carga uniforme.

Se han aplicado las correspondientes cargas en el agujero destinado a fijar el tirante de cercha, aplicándole la totalidad de los esfuerzos que éste le transmite. Por otra parte, se han considerado las reacciones que origina sobre el capitel la tornillería encargada de fijar la abrazadera de unión del arco al capitel $\left(R_{1 \text { Y.z. } x}: 0,21,-0,26,0,38\right.$; $\left.R_{2 Y . Z, X}: 0,21,-0,19,0,38 \mathrm{kN}\right)$.

Dada la ausencia de simetría alguna en la articulación, únicamente se han aplicado condiciones de coacción en las superficies de contacto del capitel con respecto al pilar, efectuándose en los tres grados de libertad.

Se procede con la simulación numérica y el análisis de resultados, finalizando la investigación con el estudio de las mejoras obtenidas con las implementaciones realizadas, verificando así su validez.

\subsection{Modelización por elementos finitos}

La simulación numérica de la estructura de invernadero se realiza con el programa ROBOT MILLENNIUM (36), mientras que el análisis de los capiteles se desarrolla con la aplicación RAM-SERIES Compass (37), atendiendo al procedimiento descrito en los apartados anteriores.

Dada la geometría irregular de los capiteles estudiados, ha sido necesario analizarlos
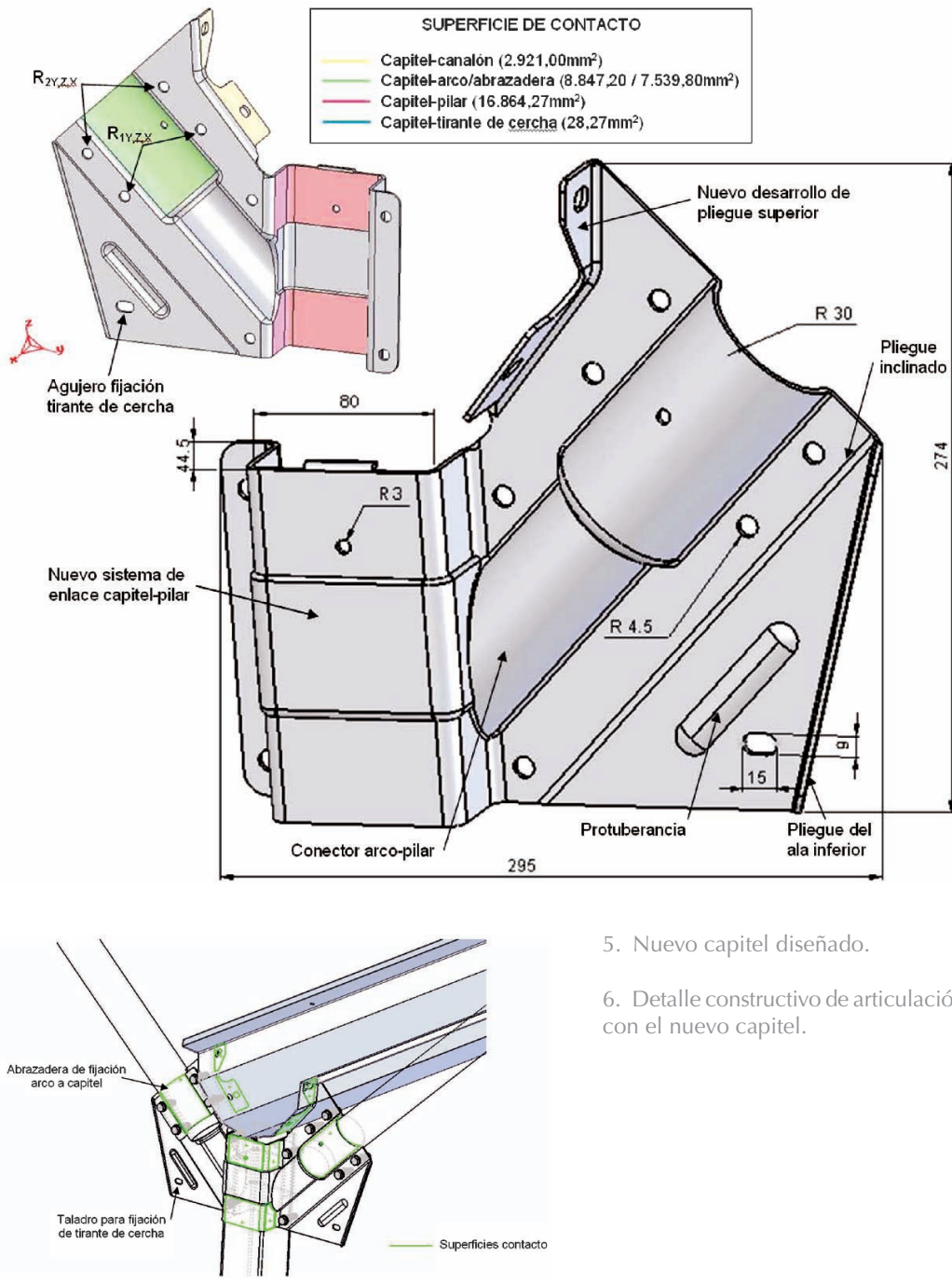

5. Nuevo capitel diseñado.

6. Detalle constructivo de articulación con el nuevo capitel.

mediante la teoría general de la elasticidad en tres dimensiones, formulando los elementos finitos como elementos tetraédricos de cuatro nodos con tres grados de libertad: desplaza- 
miento en las direcciones nodales $x, y, z$. Se ha pronosticado el empleo de mallas más tupidas en las zonas donde se preveían gradientes de tensiones elevados para minimizar los errores cometidos. Tras el desarrollo de análisis preliminares, se ha decidido emplear mallas constituidas por elementos de $10 \mathrm{~mm}$; con 14.533 nodos y 46.320 elementos tetraédricos en el capitel base y 11.575 nodos y 34.905 elementos tetraédricos en el nuevo capitel. Las características de los materiales empleados, tanto en la estructura de invernadero como en los capiteles, fueron las correspondientes a la clase resistente considerada como acero S-235 (Límite elástico $235 \mathrm{MPa}$; Módulo de Elasticidad $210 \mathrm{kN} / \mathrm{mm}^{2}$, Módulo de Elasticidad Transversal $81 \mathrm{kN} / \mathrm{mm}^{2}$, Coeficiente de Poisson 0,3 y densidad $7.850 \mathrm{~kg} / \mathrm{m}^{3}$ ).

\section{Tabla 1}

Casos de carga estudiados

\begin{tabular}{|c|c|}
\hline Acción & EN 13031-1 \\
\hline Permanente & $\begin{array}{r}\text { Estimación automática por el programa atendiendo } \\
\text { perfilería empleada } 62,2 \mathrm{~N} \mathrm{~m}^{-2}\end{array}$ \\
\hline Viento & $\begin{array}{c}\text { Velocidad característica } 118,7 \mathrm{~km} \mathrm{~h}^{-1} ; \\
\text { Presión característica } 679,5 \text { Pa según (33) } \\
\text { Coeficientes aerodinámicos para Presión neta según } \\
\text { anexo B }\end{array}$ \\
\hline Nieve & $\begin{array}{c}\text { Coeficiente térmico 1 } \\
\text { Carga de nieve 0,144 } \mathrm{kN} \mathrm{m}^{-2} \text { según }(34) \\
\text { Coeficientes de forma según anexo C }\end{array}$ \\
\hline Productos & Cosechas como tomates y pepinos: $0,15 \mathrm{kN} \mathrm{m}^{-2}$ \\
\hline
\end{tabular}

Tabla 2

Combinación de acciones estudiada.

\begin{tabular}{|c|c|}
\hline Hipótesis & $\begin{array}{l}\text { Combinación de acciones, } \\
\text { coeficientes parciales y de combinación }\end{array}$ \\
\hline a1 & $\begin{array}{c}\text { Peso propio } \cdot 1,2+\text { Viento } \cdot 1,2+\text { Nieve } \cdot 1,2 \cdot 0,5+ \\
\text { Cultivo } \cdot 1,2 \cdot 1,0\end{array}$ \\
\hline a2 & Peso propio $\cdot 1,2+$ Nieve $\cdot 1,2+$ Cultivo $\cdot 1,2 \cdot 1,0$ \\
\hline a3 & Peso propio $\cdot 1,2+$ Viento $\cdot 1,2 \cdot 1,0+$ Cultivo $\cdot 1,2$ \\
\hline b1 & Peso propio $\cdot 1,2+$ Viento $\cdot 1,2$ \\
\hline
\end{tabular}

Tabla 3

Esfuerzos máximos alcanzados por los elementos constructivos enlazados por el nudo capitel más limitativo, para la combinación de cargas a1 según EN 13031-1

\begin{tabular}{|c|c|c|c|c|}
\hline \multicolumn{5}{|c|}{ Esfuerzos y momentos combinación a1 } \\
\hline Elemento & Arco & Barra de cultivo & Canalón & Pilar \\
\hline $\boldsymbol{N}(\boldsymbol{k N})$ & 8,03 & $-4,82$ & $-3,45$ & 17,04 \\
\hline $\boldsymbol{T}_{\boldsymbol{y} \text {-vertical }}(\boldsymbol{k N})$ & $-0,01$ & 0,00 & 3,38 & 0,00 \\
\hline $\boldsymbol{T}_{\mathbf{z} \text {-Horizontal }}(\boldsymbol{k N})$ & $-1,15$ & $-0,68$ & $-0,04$ & $-0,30$ \\
\hline
\end{tabular}

\section{RESULTADOS}

\subsection{Análisis M.E.F. de estructura de invernadero industrial}

La Tabla 1 muestra los valores característicos de las acciones consideradas en el cálculo, habiendo sido empleado para su determinación el período mínimo de referencia y la probabilidad anual de excedencia del invernadero estudiado.

La Tabla 2 muestra las combinaciones de acciones, los valores numéricos de los coeficientes parciales y los coeficientes de combinación empleados.

La combinación de carga más desfavorable resulta ser la hipótesis a1, actuando las cargas a sobrepresión. La Tabla 3 muestra los esfuerzos axiales y cortantes registrados por los elementos ensamblados por los nudos capitel más limitativos, ubicados en la Figura 3.

\subsection{Análisis M.E.F. del capitel base}

Seguidamente, se muestran los resultados de distribución de tensiones y de deformación del nudo capitel modelado.

\section{Estudio de distribución de tensiones.}

La Figura 7 muestra la distribución de tensiones de Von Mises del capitel base.

Las tensiones máximas se alcanzan en las zonas designadas como 1, registrándose valores de $501,6 \mathrm{~N} / \mathrm{mm}^{2}$. Además se hace perceptible la presencia de otras regiones, tales como el ala superior del capitel (2) y la región comprendida entre el arco y el pilar (3), donde las tensiones superan los $300 \mathrm{~N} / \mathrm{mm}^{2}$. En todo caso, dichas tensiones superan la resistencia dúctil del acero empleado, $235 \mathrm{~N} / \mathrm{mm}^{2}$.

La simulación numérica del capitel base corrobora las patologías estructurales detectadas en los capiteles muestreados en campo. Así, las fisuras y/o deformaciones perceptibles en las zonas designadas como 1 quedan justificadas por el comportamiento plástico del material en dicha región.

Como consecuencia de los resultados, se plantea realizar las siguientes propuestas de mejora:

- Diseño de un conector entre el arco-pilar para favorecer entre ellos la transferencia de esfuerzos, evitando así las concentraciones de tensiones registradas en 1 .

- Nuevo diseño del pliegue superior para evitar concentración de tensiones excesivas en la zona designada como 2. 
Estudio de deformación.

La Figura 8 muestra la deformación del capitel base.

La deformación máxima se alcanza en la zona de enlace del arco con el capitel, en su extremo, registrando valores máximos de 21,3 mm, dicha deformación conlleva flechas estimadas en cumbrera de $113 \mathrm{~mm}$.

Dada la clase de invernadero estudiada, invernadero de clase B, la norma europea (1) no prescribe limitaciones en los desplazamientos de la estructura como consecuencia de la acción de las cargas. No obstante, las deformaciones que presenta el capitel estudiado son elevadas, siendo conveniente reducirlas.

Con los resultados obtenidos, se plantean las siguientes propuestas de mejora:

- Aumento del desarrollo del capitel en el plano de trabajo mediante diseño de un sistema de acartelamiento inferior constituido por: pliegue inclinado, protuberancia y pliegue del ala inferior.

- Refuerzo del sistema de enlace capitel-pilar mediante su rediseño.

\subsection{Análisis M.E.F. del nuevo capitel diseñado}

La Figura 5 muestra el nuevo capitel diseñado usando las propuestas de mejora establecidas en los apartados anteriores.

\section{Estudio de distribución de tensiones.}

La Figura 9 muestra la distribución de tensiones de Von Mises del nuevo capitel diseñado.

Las tensiones máximas se producen en la zona designada como 4. Se alcanzan valores de $152,4 \mathrm{~N} / \mathrm{mm}^{2}$, representando el $64,85 \%$ de las máximas admisibles por el acero empleado, $235 \mathrm{~N} / \mathrm{mm}^{2}$. En las restantes zonas, las tensiones son inferiores a $100 \mathrm{~N} / \mathrm{mm}^{2}$.

\section{Estudio de deformación.}

La Figura 10 muestra la deformación registrada por el nuevo capitel.

Las deformaciones máximas se producen en el extremo del pliegue del ala superior del capitel, alcanzando valores de 15,8 mm. Se ha de mencionar que las deformaciones se producen en el plano perpendicular al capitel, plano XZ, siendo prácticamente nula la deformación existente en el plano de trabajo del capitel, plano YZ.

\section{DISCUSIÓN}

Complementando las investigaciones realizadas por Lim et. al. (2002) (30), Li et. al. (2004) (26), Kim et. al. (2008) (27) y Villar et. al. (2009) (28), se ha diseñado un nuevo capitel para invernaderos multitúnel compatible con el procedimiento constructivo de invernadero de cubierta elevable propuesto Carreño (2005) (20) y Galera et. al. (2007) (21).
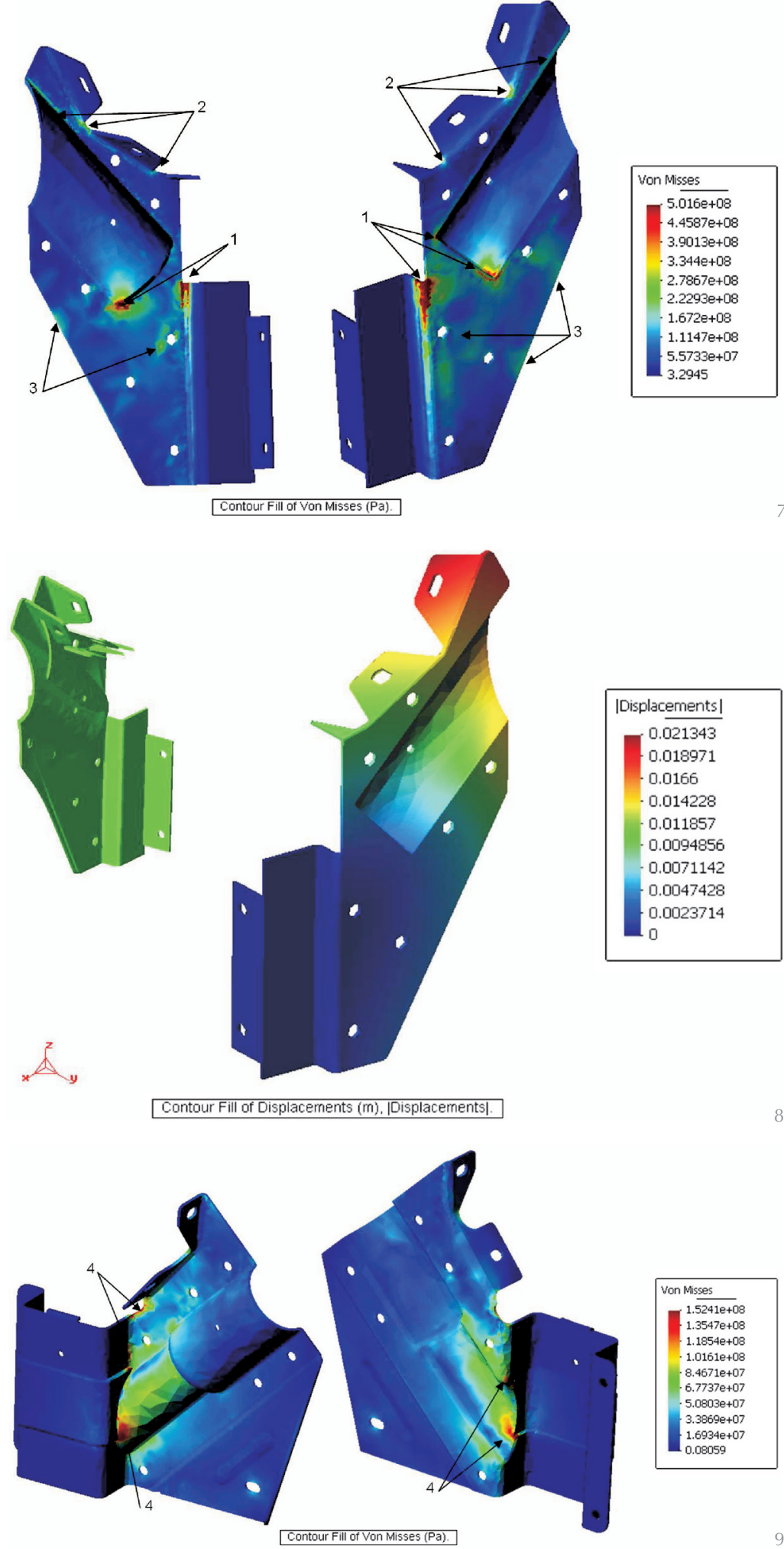


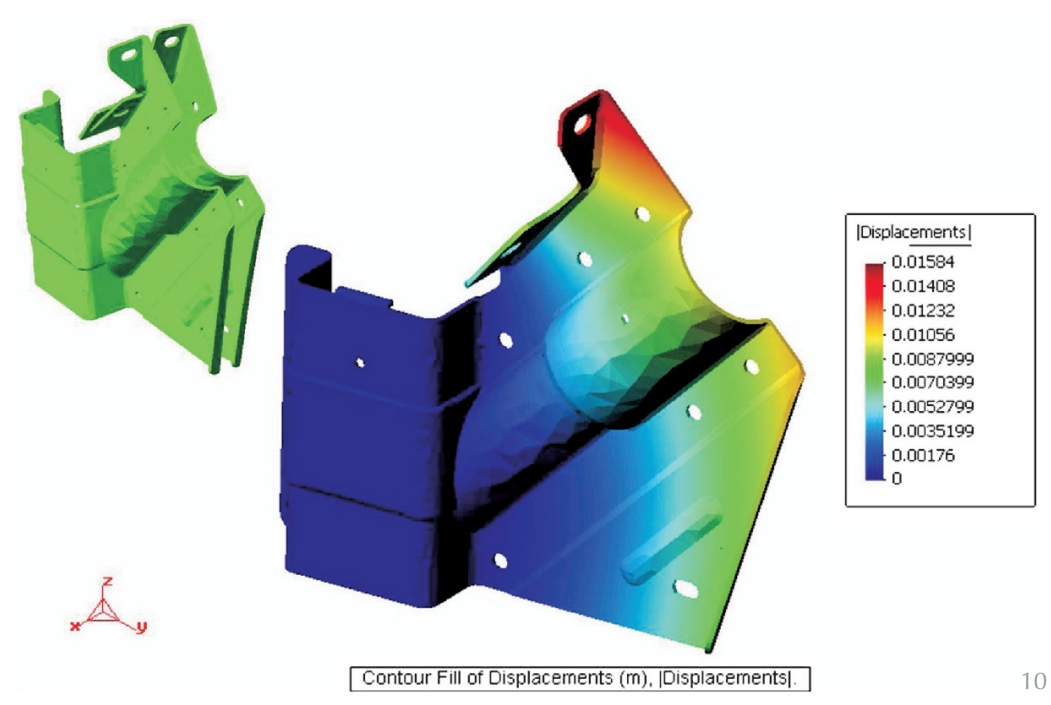

10. Deformación del nuevo capitel.
Para el conocimiento de las fuerzas que actúan sobre los capiteles, de forma previa ha sido necesario el análisis M.E.F. del comportamiento estructural de un invernadero industrial, para lo cual se ha atendido a las recomendaciones de Waaijenberg et. al. (2006) (10), desarrollando los cálculos según lo prescrito en EN 13031-1 (1) y normativa complementaria UNE 76209IN (33), ENV 1991-2-4 (34) y ENV 1991-2-3 (35).

De esta forma ha sido posible seleccionar el nudo capitel más desfavorable para la hipótesis combinatoria más restrictiva y conocer los esfuerzos a los que está sometido. Para la geometría y la ubicación de invernadero estudiada, la combinación de cálculo más desfavorable para el diseño y dimensionado del nudo capitel es la combinación de cargas a1 según EN 13031-1 (1).

El análisis M.E.F. del capitel base, muestra la presencia de regiones donde las tensiones registradas superan la resistencia dúctil del acero empleado, alcanzándose valores máximos de 501,6 N/ $\mathrm{mm}^{2}$. De esta forma, se corroboran analíticamente los defectos detectados visualmente en forma de fisuras y/o deformaciones en los capiteles muestreados en campo. Por otra parte, el análisis M.E.F. realizado ha permitido detectar nuevas regiones infradimensionadas, el ala superior del capitel y la zona situada bajo el arco, donde las tensiones resultan también excesivas por el material empleado. Por otro lado, las defor- maciones máximas alcanzadas se cifran en 21,3 mm, las cuales conllevan flechas estimadas en cumbrera de $113 \mathrm{~mm}$. Pese a que dada la clase de invernadero no es necesario comprobación a Estado Límite de Servicio, se considera elevada la deformación sufrida. Del análisis de deficiencias del capitel base se plantean las siguientes propuestas de mejora; diseño de un conector entre el arco-pilar, rediseño del pliegue superior, aumento del desarrollo del capitel en el plano de trabajo con el diseño de un sistema de acartelamiento inferior y un nuevo diseño del sistema de enlace capitel-pilar.

La simulación numérica del nuevo capitel ha demostrado la validez de las implementaciones realizadas. La disposición del conector arco-pilar junto con el sistema de acartelamiento inferior diseñado consigue reducir las tensiones hasta valores holgadamente inferiores a las admisibles por el acero empleado, con valores máximos de 152,4 N/mm². En cuanto a deformación, nuevamente el aumento del desarrollo en el plano de trabajo con el sistema de acartelamiento inferior y el nuevo sistema de enlace capitel-pilar, logran reducir las deformaciones en el plano de trabajo del capitel hasta valores prácticamente nulos, controlando así las elevadas flexiones que el uso del capitel base conllevaba en cumbrera de arcos. Dada la asimetría de la articulación, las deformaciones máximas se producen en el plano perpendicular al de trabajo del capitel, alcanzándose unos valores máximos de 15,8 $\mathrm{mm}$ en el extremo del pliegue superior. En todo caso, dada la ausencia de fenómeno alguno de deformación plástica en el material empleado, dichas deformaciones resultan aceptables.

De esta forma, se verifica que el desarrollo, material y dimensiones del nuevo capitel diseñado solventan las deficiencias estructurales del capitel base en materia de tensión y deformación. A su vez, se prevé que el empleo del nuevo capitel reduzca los tiempos de montaje de la estructura, al reducir el número de piezas a ensamblar en altura así como el número de tornillos a disponer (capitel base 26, nuevo capitel 18).

\section{AGRADECIMIENTOS}

Los autores desean expresar su agradecimiento a Novedades Agrícolas S.A. por financiar el presente trabajo mediante proyecto de investigación APR System Project.

\section{BIBLIOGRAFÍA}

(1) prEN 13031-1. "Greenhouses: design and construction. Part 1: Commercial production greenhouses", European Committee for standardization CEN. p. 105, Brussels, Belgium. 2002.

(2) Von Elsner, B.; Briassoulis, D.; Waaijenberg, D.; Mistriotis, A.; Von Zabeltitz, C.; Gratraud, J.; Russo, G.; Suay-Cortes, R.: "Review of structural and functional characteristics of greenhouses in European Union countries, part I: design requirements", Journal of Agricultural Engineering Research, Vol. 75 nº 1 (2000), pp. 1-16. doi:10.1006/jaer.1999.0502. 
(3) Castilla, N.; Hernández, J.: "The plastic greenhouse industry of Spain", Chronica Horticulturae, Vol. 45 no 3 (2000), pp. 15-20.

(4) Von Elsner, B.; Briassoulis, D.; Waaijenberg, D.; Mistriotis, A.; Von Zabeltitz, C.; Gratraud, J.; Russo, G.; Suay-Cortes, R.: "Review of Structural and Functional Characteristics of Greenhouses in European Union Countries, Part II:Typical Designs", Journal of Agricultural Engineering Research, Vol. 75 no 2 (2000), pp. 111-126, doi:10.1006/jaer.1999.0512.

(5) Sanjuán, J. F.: "Detección de la superficie invernada en la provincia de Almería a través de IMÁG",Si es un libro, falta el nombre del mismo p. 68, Ed. FIAPA, Almería, Spain, 2007.

(6) Callejón, A. J.; Carreño, A.; Sánchez-Hermosilla, J.; Pérez, J.: "Evaluación de impacto ambiental de centro de transformación y gestión de residuos sólidos agrícolas en la provincia de Almería (España)", Informes de la Construcción, Vol. 62 n 518 (2010), pp. 79-93, doi: 10.3989/ic.08028.

(7) Callejon-Ferre, A. J.; Manzano-Agugliaro, F.; Díaz-Pérez, M.; Carreño-Ortega A.; PérezAlonso J.: "Effect of shading with aluminised screens on fruit production and quality in tomato (Solanum lycopersicum L.) under greenhouse conditions", Spanish Journal of Agricultural Research Vol. 7 n 1 (2009), pp. 41-49.

(8) López-Hernández, J. C.; Pérez-Parra, J.: "Evolución de las estructuras de invernadero", Plasticulture, Vol. 125 (2006), pp. 8-17.

(9) Iribarne, L.; Ayala, R.; Torres, J. A.: "A DPS-based system modelling method for 3Dstructures simulation in manufacturing processes", Simulation modelling practice and theory, Vol. 17 no 5 (2009), pp. 935-954. doi:10.1016/j.simpat.2009.03.001.

(10) Waaijenberg, D.: "Design, construction and maintenance of greenhouse structures". Acta Horticulturae, Vol. 710 (2006), pp. 31-42.

(11) Carreño, A.; Pérez, J.; Callejón, A.; Vázquez, J.; López, G.; Sánchez-Hermosilla, J.: "Analysis of the structural behaviour of European standardized multispan type greenhouses in the context of exporting", International conference on agricultural engineering (Ageng 2008), Crete, Greece, 2008.

(12) Callejón, A.; Carreño, A.; Pérez, J.; Vázquez, J.; Galera, L.: "Structural improvements in multispan greenhouses for verification the European standard UNE-EN 13031-1", XXXIII CIOSTA-CIGR V Conference 2009: Technology and management to ensure sustainable agriculture, agro-systems, forestry and safety. Reggio Calabria, Italy, 2009.

(13) Castellano, S.; Giuliano, V.; Scarascia-Mugnozza, G.: "Collapse full scale test on a steel greenhouse tunnel structure", Rivista di ingegneria agrarian, n 3 (2002), pp. 49-58.

(14) Yekutieli, O.; Dubinski, L.; Kleinmann, I.: "Analysis of forces acting due to strong wind loads on structure and cover of a walk-in tunnel", International conference and BritishIsraeli workshop on greenhouse technologies, Vol. 443 (1997), pp. 53-61.

(15) Mistriotis, A.; Briassoulis, D.: "Numerical estimation of the internal and external aerodynamic coefficients of a tunnel greenhouse structure with openings", Computers and Electronics in Agriculture, Vol. 34 (2002), pp. 191-205. doi:10.1016/S0168-1699 (01) 00187-9.

(16) Robertson, A. P.; Roux, Ph.; Gratraud, J.; Scarascia, G.; Castellano, S.; Dufresne de Virel, M.; Palier, P.: "Wind pressures on permeably and impermeably-clad structures", Journal of Wind Engineering and Industrial Aerodynamics, Vol. 90 n 4-5 (2002), pp. 461-474. doi:10.1016/S0167-6105(01)00210-0.

(17) Briassoulis, D.: "Mechanical design requirements for low tunnel biodegradable and conventional films", Biosystem Engineering, Vol. 87 no 2 (2004), pp. 209-223. doi:10.1016/j. biosystemseng.2003.10.013.

(18) Pérez, J.; Carreño, A.; Callejón, A.; Vázquez, F. J.; Sánchez-Hermosilla, J.; Pérez, J. J.; Galera, L.: "Identification of Factors Determining the Preventive Action of the Greenhouses Construction Companies in the Southeast of Spain", XXXIII CIOSTA - CIGRV Conference 2009: Technology and management to ensure sustainable agriculture, agro-systems, forestry and safety. Reggio Calabria, Italy, 2009.

(19) Carreño, A.; Pérez, J.; Vázquez, F. J.; Callejón, A.: "La siniestralidad laboral en la construcción de invernaderos". Agrícola Vergel. Vol. 28 n 332 (2009), pp. 440-443.

(20) Carreño, A.: "Reducción de la siniestralidad laboral en la construcción de invernaderos tipo multitúnel mediante la implementación de un nuevo procedimiento constructive" Servicio de Publicaciones de la Universidad de Almería, p. 453, Almería, 2005.

(21) Galera, L.; Carreño, A.; Pérez, J.: "Método constructivo para invernaderos y dispositivo para la puesta en práctica del mismo", Referencia patente: P200402287, 2007.

(22) Behm, M.: "Linking construction fatalities to the design for construction safety concept", Safety Science, Vol. 43 no 8 (2005), pp. 589-611. doi:10.1016/j.ssci.2005.04.002.

(23) Van Gorp, A.: 2007. "Ethical issues in engineering design processes; regulative frameworks for safety and sustainability", Design Studies, 28 n 2 (2006), pp. 117-131. doi:10.1016/j. destud.2006.11.002. 
(24) Gambatese, J. A.; Behm, M., Rajendran, S.: "Design's role in construction accident causality and prevention: Perspectives from an expert panel". Safety Science, Vol. 46 no 8 (2007), pp. 675-691. doi:10.1016/j.ssci.2007.06.010.

(25) Toole, T. M.; Gambatese, J.: "The trajectories of Construction Prevention through Design", Journal of Safety Research, 39 n 2, pp. 225-230. doi:10.1016/j.jsr.2008.02.026.

(26) Li, Y. J.; Juan, W.; Maoai, C.; Shen, X. Q.: "Finite element analysis of residual stress in the welded zone of a high strength steel", Bulletin of materials science, Vol. $27 \mathrm{n}^{\circ} 2$ (2004), pp. 127-132.

(27) Kim, T.; Kuwamura, H.; Cho, T.; Shin, S.; Kim, S.; Lee, Y.: "Investigation on mechanical behaviors for bolted connections in carbon steel and in stainless steel using FEM", ISIJ international, Vol. 48 n $^{\circ} 6$ (2008), pp. 851-860.

(28) Villar, J. R.; Guaita, M.; Vidal, P.; Argüelles Bustillo, R.: "Numerical simulation of framed joints in sawn-timber roof trusses", Spanish Journal of Agricultural Research, Vol. 6 n 4 (2008), pp. 508-520.

(29) Makelainen, P.; Kesti, J.: "Advanced method for lightweight steel joining", Journal of constructional steel research, Vol. 49 no 2 (1999), pp. 107-116. doi:10.1016/S0143974X(98)00210-7.

(30) Lim, J. B. P.; Nethercot, D. A.: "F. E.-assisted design of the eaves bracket of a cold-formed steel portal frame", Steel \& composite structures, Vol. $2 n^{\circ} 6$ (2002), pp. 411-428.

(31) Toma, A. W.: "Developments in connections in cold-formed building structures and design specifications", Prog. Eng. Mater Struct, Vol. 5 (2003), pp. 145-152. doi:10.1016/ S0143-974X(99)00089-9.

(32) Fernández, C.; Pérez, J.: "Caracterización de los invernaderos de la provincia de Almería", Cajamar, p. 24, Almería, Spain, 2004.

(33) UNE 76209IN. "Wind actions in greenhouses for commercial production". AENOR. p. 11, Madrid, Spain, 2002.

(34) ENV 1991-2-4. Eurocode 1: "Basis of design and actions on structures. Part 2-4: Action on structures. Wind action", European Committee for standardization CEN, p. 138, Brussels, Belgium, 1998.

(35) ENV 1991-2-3. Eurocode 1: "Basis of design and actions on structures. Part 2.3: Action on structures. Snow loads", European Committee for standardization CEN. p. 60, Brussels, Belgium, 1998.

(36) http://usa.autodesk.com/adsk/servlet/pc/index?sitelD=123112\&id=11818169.

(37) http://www.compassis.com/compass. 